\title{
Fibrilación auricular paroxística en ataque cerebro-vascular criptogénico
}

\section{Paroxysmal atrial fibrillation in cryptogenic cerebrovascular accident}

\author{
Javier Torres-Zafra
}

Grupo ataque cerebro-vascular, Clínica Shaio, Bogotá, Colombia

Recibido el 3 de octubre de 2016; aceptado el 6 de octubre de 2016 Disponible en Internet el 8 de noviembre de 2016

\section{Introducción}

Para algunos el término criptogénico no es más que una apología a la ignorancia sobre los mecanismos fisiopatológicos del ataque cerebrovascular ${ }^{1}$. Sin embargo, en las últimas dos décadas el adelanto en imágenes diagnósticas cerebrales, en cardiología no invasiva, en monitorización del ritmo cardiaco y en estudios epidemiológicos ha logrado una mejor precisión de la causa del ataque cerebro-vascular de etiología desconocida.

Por su alta incidencia y porque la identificación de su etiología modifica la conducta terapéutica mediante un tratamiento específico, el ataque cerebro-vascular criptogénico continúa atrayendo la atención del personal médico.

La incidencia reportada de ataque cerebro-vascular criptogénico varía de acuerdo con la definición, la edad del paciente y cuán profunda haya sido la investigación clínica. Su incidencia en adultos, en estudios poblacionales y registros hospitalarios, es del 20 al $40 \%$ y se ha mantenido constante a través del tiempo ${ }^{2}$. La mortalidad a dos años en el grupo de causa no establecida o criptogénico alcanzó el $39 \%^{3}$.

Los mecanismos causantes del ataque cerebro-vascular criptogénico pueden dividirse en tres grandes categorías: sospecha de hipercoagulabilidad con y sin foramen ovale persistente; enfermedad aterosclerótica de la aorta o de los vasos supraaórticos; y arritmias ocultas ${ }^{4}$.

Correo electrónico: javier.torres@shaio.org
Esta revisión se centrará en el rol de las arritmias cardiacas en los pacientes con ataque cerebro-vascular criptogénico.

\section{Evolución del concepto de ataque cerebro-vascular criptogénico}

Se considera que un ataque cerebro-vascular es criptogénico cuando no se identifica causa alguna, pero esta condición depende de la extensión y calidad de la investigación etiológica y del conocimiento actual de los mecanismos del mismo ${ }^{2}$.

Se definió en el sistema de clasificación TOAST (The Trial of Org in Acute Stroke Treatment $)^{5}$ como aquel infarto no atribuido a una causa definida de aterosclerosis de gran arteria, cardioembolia o enfermedad de pequeño vaso en presencia de: extensa evaluación cardiaca, vascular, hematológica y serológica, evaluación incompleta y cuando se presente más de una causa (v. gr. fibrilación auricular y estenosis de la arteria carótida interna con repercusión hemodinámica). Este sistema de clasificación sobreestima la proporción de ataque cerebro-vascular criptogénico y posee una baja concordancia interobservador para esta modalidad (к 0,40$)^{6}$.

En el método de clasificación causal para ataque cerebro-vascular isquémico agudo, conocido con el acrónimo SSS-TOAST ${ }^{7}$, el ataque cerebro-vascular de origen indeterminado se divide en: embolia criptogénica, otros criptogénicos y los no clasificados cuando se identifica más de un mecanismo probable. La embolia criptogénica a su vez 
Tabla 1 Criterios para el diagnóstico de ataque cerebro-vascular embólico de fuente no determinada (ESUS)

- Ataque cerebro-vascular detectado por TAC o IRM no lacunar

- Ausencia de aterosclerosis intra o extracraneana con estenosis mayor al 50\% de la luz en la arteria que suple el área de isquemia.

- No fuente cardioembólica de riesgo mayor.

- No identificación de causa específica para el ataque cerebro-vascular (v. gr. arteritis, disección, migraña/vasoespasmo, drogas o medicamentos).

TAC: tomografía axial computarizada. IRM: imagen por resonancia magnética.

Tabla 2 Propuesta de aproximación diagnóstica en ataque cerebro-vascular embólico de fuente no determinada (ESUS)

- TAC o IRM cerebral

- ECG de 12 derivaciones

- Ecocardiograma transtorácico

- Holter (ECG 24 horas)

- Imágenes tanto de arterias intracraneanas como extracraneanas que suplen el área de isquemia (cateterismo, angiorresonancia, angio-TAC, o Doppler y dúplex de vasos de cuello más Doppler transcraneal)

ECG: electrocardiograma.

se definió como: evidencia angiográfica de una amputación abrupta consecuente con un coágulo sanguíneo en arterias intracraneanas por lo demás angiográficamente normales, o evidencia por imágenes de recanalización completa en una arteria previamente ocluida, o la presencia de infartos cerebrales agudos múltiples ocurridos en estrecha relación temporal sin anormalidades detectadas en los vasos involucrados.

El sistema de clasificación fenotípico para ataque cerebro-vascular isquémico $\mathrm{ASCO}^{8}$ (su sigla en inglés por Atherosclerosis, Small vessel disease, Cardiac source y Other cause), no tuvo en cuenta el ataque cerebro-vascular criptogénico por considerarlo un diagnóstico de exclusión $\left(\mathrm{A}_{0} \mathrm{~S}_{0} \mathrm{C}_{0} \mathrm{O}_{0}\right)$.

Finalmente, el término ESUS (Embolic Stroke of Undetermined Source) ${ }^{9}$ o ataque cerebro-vascular embólico de fuente no determinada apareció como un constructo para posicionarlo de manera pragmática como una entidad terapéuticamente relevante y así tratar estos pacientes con anticoagulantes para disminuir la recurrencia.

Se define ataque cerebro-vascular embólico de fuente no determinada (ESUS) como un infarto cerebral no lacunar, sin estenosis arterial proximal o fuente cardioembólica con una clara indicación para anticoagulación (tabla 1), que haya cumplido con una pesquisa etiológica adecuada (tabla 2).

\section{Las huellas del ataque cerebro-vascular embólico}

Cumplida la investigación etiológica recomendada en la tabla 2 sin establecer la causa del ataque cerebro-vascular, la sospecha de ataque cerebro-vascular embólico se sustenta en el patrón de la lesión en imágenes cerebrales y en otras características del paciente.

Se debe analizar la resonancia magnética cerebral en el siguiente orden ${ }^{10}$ :

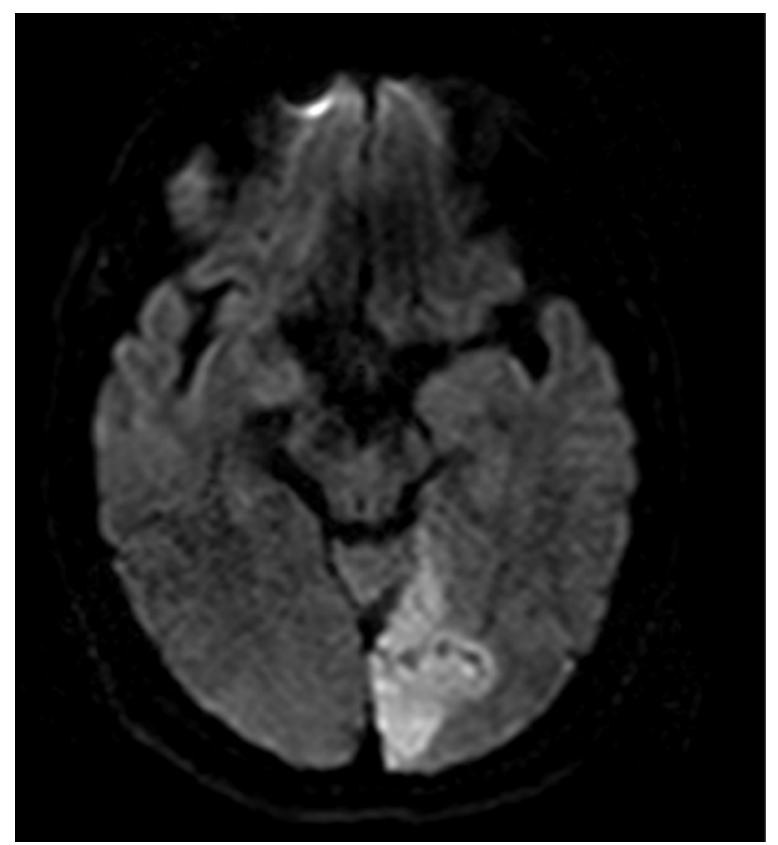

Figura 1 IRM de paciente con infarto agudo de la arteria cerebral posterior izquierda secundario a fibrilación auricular con hiperintensidad en DWI.

1. Patrón de difusión o DWI (Diffusion Weighted Imaging): embolia versus profundo y grande versus pequeño disperso.

2. Distribución del infarto en DWI: más de un territorio vascular comprometido.

3. Historia de ataques cerebro-vasculares anteriores en FLAIR (Fluid Attenuated Inversion Recovery).

Las lesiones cortico-subcorticales y las bilaterales múltiples en la circulación anterior y posterior, se asocian con cardioembolia, mientras que las lesiones unilaterales múltiples en la circulación anterior se asocian con embolia arterio-arterial por aterosclerosis o patología disecante.

Las grandes lesiones corticales en uno o varios territorios en la DWI con diferentes edades en FLAIR, sugieren embolia por fibrilación auricular (fig. 1).

Las lesiones pequeñas y dispersas en múltiples territorios en DWI sugieren coagulopatía relacionada con cáncer, embolia paradójica o embolia aortogénica ${ }^{11}$.

Los infartos cerebrales por embolia paradójica en casos de foramen ovale persistente se ubican principalmente en territorio posterior ${ }^{12}$. 
En un estudio de 131 pacientes con ataque cerebrovascular criptogénico el $58 \%$ de ellos tuvieron perfil genético de cardioembolia ${ }^{13}$. Otro estudio ${ }^{14}$ comparó el subgrupo de pacientes clasificados en el TOAST como de causa indeterminada por tener más de una posible etiología con aquellos de causa no conocida y se estableció en el primero de ellos mayor promedio de edad, mayores puntajes en la escala de ataque cerebro-vascular de los Institutos Nacionales de Salud (NIHSS), así como mayor proporción de diabetes mellitus tipo 2, frecuencia de falla cardiaca y de fibrilación auricular, estancia hospitalaria y peor pronóstico funcional.

\section{Fibrilación auricular paroxística en ataque cerebro-vascular criptogénico}

La fibrilación auricular puede detectarse en los pacientes con ataque cerebro-vascular criptogénico o ataque isquémico transitorio de causa no definida, incluso años después del evento vascular cerebral índice. La fibrilación auricular aumenta significativamente el riesgo de recurrencia de ataque cerebro-vascular y el tratamiento anticoagulante con warfarina reduce en un $64 \%$ el riesgo de ataque cerebrovascular comparado con placebo y hasta en un $39 \%$ si se compara con Aspirina ${ }^{15}$. Los anticoagulantes directos son tan eficaces o incluso superiores a la warfarina en la prevención secundaria ${ }^{16}$. Por tanto, el esfuerzo por detectar arritmias cardiacas ocultas generadoras de embolia está garantizado.

La fibrilación auricular paroxística es usualmente asintomática y transitoria lo cual dificulta su detección. Cuando esta se detecta por primera vez después de un ataque cerebro-vascular criptogénico, aumenta el riesgo de recurrencia del ataque aún si se compara con el grupo de pacientes en los que la fibrilación auricular era conocida ${ }^{17}$.

El término "fibrilación auricular silenciosa" ha surgido recientemente para describir arritmias auriculares no detectables clínicamente, pero sí por medio de dispositivos electrónicos cardiacos implantables ${ }^{18}$. Si embargo, el porqué la "fibrilación auricular silenciosa" aumenta el riesgo de recurrencia o de aparición del ataque cerebro-vascular isquémico, aún no se ha establecido por completo.

El CRYSTAL AF fue un estudio prospectivo, multicéntrico y aleatorizado en el que se comparó la monitorización tradicional con los dispositivos cardiacos implantables (ICM Insertable Cardiac Monitor) ${ }^{19}$. Los pacientes eran mayores de 40 años y en los últimos 90 días debían haber tenido un ataque cerebrovascular o ataque isquémico transitorio. La pesquisa etiológica incluyó electrocardiograma (EKG) de 12 derivaciones, ecocardiograma transesofágico, angio-TAC o angiorresonancia intra- y extracraneana y Holter de 24 horas, adicionalmente, en los pacientes menores de 55 años se investigó trombofilia. El desenlace primario fue el tiempo para detectar el primer episodio de fibrilación auricular durante los primeros seis meses de monitorización y los secundarios incluyeron el tiempo para la primera detección de fibrilación auricular durante los primeros 12 meses de seguimiento, ataque cerebrovascular recurrente o ataque isquémico transitorio y cambio en el uso de anticoagulantes orales.

La fibrilación auricular se definió como aquella arritmia sin ondas $\mathrm{p}$ y con una duración mayor a 30 segundos. En el brazo de monitorización tradicional se detectó fibrilación auricular en $1,4,2$ y $3 \%$, en tanto que en el grupo de los dispositivos cardiacos insertables $8.9,12,4$ y $30 \%$ a los 6,12 y 36 meses respectivamente. A los tres años se detectó diez veces más fibrilación auricular en el grupo de dispositivos insertables.

Tres cuartas partes de los pacientes fueron asintomáticos y en un $97 \%$ de los casos tuvieron por lo menos un episodio de fibrilación auricular mayor a 6 minutos. El tiempo medio entre la aleatorización y la detección del primer episodio el grupo de dispositivos insertables a los 6 meses fue de 41 días y 84 días a los 12 meses. El ataque cerebrovascular isquémico o el ataque isquémico transitorio ocurrió en el 5,2 vs. $8,6 \%$ a los 6 meses y en 7,1 vs. $9,1 \%$ a los 12 meses en el grupo de dispositivos insertables vs. el grupo control, respectivamente. El uso de anticoagulantes fue del 10,1 vs. $4,6 \%$ a los 6 meses y de 14,7 vs. $6,0 \%$ a los 12 meses en el grupo de pacientes con dispositivos insertables vs. el grupo control respectivamente. A los 12 meses, el $97 \%$ de los pacientes en quienes se había detectado fibrilación auricular recibía anticoagulantes orales.

Por su parte, el estudio EMBRACE, publicado simultáneamente con el anterior en junio de 2014, comparó así mismo la monitorización de eventos a los 30 días (30 Days Triggering Event Recorder) con la monitorización convencional ${ }^{20}$. Se detectó fibrilación auricular en el 16,1 del grupo intervenido vs. $3,2 \%$ en el grupo control.

En una revisión sistemática y metaanálisis se estimó la proporción de pacientes con fibrilación auricular diagnosticados por primera vez después de un ataque cerebrovascular o ataque isquémico transitorio en una secuencia de cuatro fases de monitorización cardiaca ${ }^{21}$. La fase 1 incluyó el EKG tomado en el servicio de urgencias, la fase 2 (hospitalaria) incluyó EKG seriados, monitorización electrocardiográfica continua, telemetría cardiaca continua y test de Holter; la fase 3 (primer periodo ambulatorio) Holter ambulatorio y la fase 4 (segundo período ambulatorio) dispositivos cardiacos externos e implantables. En la categoría 1 se detectó fibrilación auricular en un 7,7, 5,1\% en la fase 2, 10,7\% en la fase 3 y $16,9 \%$ en la 4.

En este meta-análisis $10,6 \%$ de los pacientes no tuvieron otros estudios más allá de la fase 1 , un $10,0 \%$ no los tuvieron más allá de la fase 2 y $60 \%$ no fueron estudiados con técnicas de monitorización cardiaca prolongada (fase 4$)^{21}$.

\section{¿Cómo estudiar los pacientes con ataque cerebro-vascular criptogénico y sospecha de fibrilación auricular oculta?}

La monitorización cardiaca prolongada con dispositivos externos o implantables es costosa y de difícil acceso ¿Qué pacientes se deberían monitorizar?

No está claro aún qué subgrupos de pacientes se benefician más de la monitorización prolongada, cuánto tiempo la requieren y para quiénes es más costo-efectivo.

Los latidos auriculares prematuros frecuentes, al igual que los brotes de taquiarritmia auricular son marcadores emergentes de riesgo que pueden ayudar a identificar a los pacientes con ritmo sinusal que desarrollarán fibrilación auricular. En un subanálisis ulterior del estudio EMBRACE $^{20}$ se tomaron aquellos pacientes con actividad ectópica supraventricular aumentada y se cuantificaron los 
latidos auriculares prematuros (APB Atrial Premature Beats) en los estudios de Holter de 24 horas $^{22}$. A los 90 días la detección global de fibrilación auricular fue del 16\%, la probabilidad de detectarla en pacientes con menos de 100 APB $/ 24$ horas fue menos de $9 \%$, entre 100-499 APB/24 horas la probabilidad aumentó de 9 a 24\%, entre 500-999 APB/24 horas de 25 a $37 \%$, de $1000-1499$ APB/24 horas de 37 a $40 \%$ y de $40 \%$ más allá de 1500 APB/24 horas. Con base en lo anterior, el grupo de investigadores del estudio EMBRACE propuso un diagrama de opciones según el cual los pacientes con ataque cerebro-vascular criptogénico en quienes no se hubiera detectado fibrilación auricular deben ir a monitorización cardiaca por dos semanas si hay menos de 500 $\mathrm{APB} / 24$ horas y durante cuatro semanas cuando es mayor 500 $\mathrm{APB} / 24$ horas. Adicionalmente, si no se detecta la fibrilación auricular, se debe considerar monitorización prolongada con dispositivos externos o insertables para pacientes con más de 1.000 APB/24 horas.

Actualmente las guías AHA/ASA recomiendan monitorización del ritmo cardiaco por treinta días en los seis meses siguientes al evento índice (clase Ila; nivel de Evidencia c) (Nueva recomendación) $)^{23}$.

\section{Carga de fibrilación auricular y riesgo de ataque cerebro-vascular}

En el estudio ASSERT ${ }^{24}$ se tomaron pacientes mayores de 65 años con hipertensión arterial e implante de marcapasos bicameral por enfermedad del nodo o enfermedad del nodo aurículo-ventricular, o un cardiodesfibrilador por cualquier indicación en las últimas ocho semanas para establecer el riesgo de un primer episodio de fibrilación auricular y de ataque cerebro-vascular. A los tres meses a un 10,1\% de los pacientes se les detectó por lo menos un episodio de taquiarritmia auricular con frecuencia mayor a 190 latidos por minuto y con una duración mayor de 6 minutos. La taquicardia auricular subclínica aumentó el riesgo de fibrilación auricular clínica con un HR (hazard ratio) de 5,56 y el de ataque cerebro-vascular con un $\mathrm{HR}$ de 2,49. Este último fue del $13 \%$, el mismo riesgo de ataque cerebro-vascular atribuido a fibrilación auricular en el estudio Framingham.

En el CRYSTAL $\mathrm{AF}^{19}$ el tiempo de fibrilación auricular fue de 30 segundos medidos en el grupo control pues el dispositivo cardiaco insertable o monitor cardiaco insertable solo detectaba a partir de dos minutos. En el estudio EMBRACE ${ }^{20}$ se tomaron también 30 segundos en la definición de fibrilación auricular en el grupo control y el dispositivo externo de cinturón detectaba hasta 2,5 minutos. Sin embargo, entre un 42 a un $85 \%$ de los casos detectados de fibrilación auricular en ataque cerebro-vascular criptogénico tienen una duración menor de 30 segundos $^{25,26}$.

\section{Relación temporal entre fibrilación auricular paroxística y ataque cerebro-vascular}

Con base en los datos actuales no parece existir una relación temporal próxima entre las arritmias auriculares detectadas en los dispositivos y la aparición de ataque cerebro-vascular, aunque los pacientes con episodios de alta frecuencia auricular (AHREs Atrial High Rate Episodes) tienen mayor riesgo de padecerlo ${ }^{18}$. En el estudio TRENDS ${ }^{27}$ un 73\%, en el ensayo ASSERT $^{28}$ un $92 \%$ y en el IMPACT ${ }^{29}$ un $94 \%$ de los pacientes no tuvieron fibrilación auricular en los 30 días previos al evento trombo-embólico.

\section{Factores de riesgo para ataque cerebro-vascular independientes de la fibrilación auricular paroxística}

La evidencia actual lleva a pensar que un tipo de cardiopatía auricular aumenta el riesgo de recurrencia de ataque cerebro-vascular en el grupo de pacientes con la forma criptogénica independiente que haya o no fibrilación auricular.

En un estudio piloto se analizaron 159 pacientes de los cuales 40 tenían ataque cerebrovascular criptogénico según los criterios del TOAST. Se definió cardiopatía auricular cuando había por lo menos uno de los siguientes biomarcadores:

1. NT ProBNP (Amino Terminal Pro-B-Type Natriuretic Peptide) con punto de corte de $250 \mathrm{pg} / \mathrm{ml}$

2. Fuerza terminal de la onda $\mathrm{p}$ en V1 (PTFV1) con umbral de 5000 uV ms, calibración EKG de $10 \mathrm{~mm} / \mathrm{mV}$ y $25 \mathrm{~mm} / \mathrm{s}$.

3. Crecimiento auricular izquierdo.

De 40 pacientes, 49\% tenían niveles elevados de NT ProBNP, 20\% PTFV1 aumentado y 5\% crecimiento auricular izquierdo. Los pacientes con cardiopatía atrial tenían alta probabilidad de ser mayores, tener hipertensión arterial, hiperlipidemia o enfermedad coronaria ${ }^{30}$.

El NT-ProBNP (Amino Terminal Pro-B-Type Natriuretic Peptide) fue medido en el suero almacenado de los pacientes reclutados en el estudio WARSS (Warfarin-Aspirine Recurrent Stroke Study) sin fibrilación auricular para comparar la efectividad relativa de la warfarina y la Aspirina en la prevención de la recurrencia del ataque cerebrovascular o de muerte en dos años basados en la concentración de NT-ProBNP. En el 5\% de los pacientes la concentración de NT-ProBNP fue mayor de $750 \mathrm{pg} / \mathrm{ml}$ y en 2 años la tasa de eventos por 100 personasaño fue de 45,9 para la Aspirina y 16,6 para la warfarina; en concentraciones menores o iguales a $750 \mathrm{pg} / \mathrm{ml}$ la tasa fue similar para ambos tratamientos, lo cual indicó el beneficio de la anticoagulación en el subgrupo con aumento de este biomarcador ${ }^{31}$.

El crecimiento auricular izquierdo moderado a severo es un factor de riesgo independiente para ataque cerebrovascular cardioembólico recurrente o ataque cerebro-vascular criptogénico ${ }^{32}$

La patogénesis de la tromboembolia en fibrilación auricular involucra disfunción endotelial, patrón anormal de flujo en el apéndice auricular, propiedades pro-coagulantes de la sangre, inflamación, factores neurohumorales y patología estructural del miocardio auricular. La característica fundamental de la patología estructural asociada con fibrilación auricular es la fibrosis ${ }^{33}$, cuyo grado empieza a ser medido mediante técnicas de realce tardío del medio de contraste en resonancia magnética cardiaca O DE-CMR (DelayedEnhancement Cardiac Magnetic Resonance). El gadolinio acorta el T1; en tejido sano este medio de contraste se disipa rápidamente, mientras que en tejido fibrótico se prolonga permitiendo estimar el grado de fibrosis. En estudios 
retrospectivos se ha podido correlacionar el grado de fibrosis con la incidencia de ataque cerebrovascular ${ }^{34}$. En el estudio DECAAF, la recurrencia de la arritmia posterior a la ablación por catéter estuvo relacionada con el porcentaje de fibrosis auricular izquierda estimada mediante realce tardío del medio de contraste en resonancia magnética ${ }^{35}$.

\section{Conclusiones}

El término ataque cerebrovascular criptogénico se aplica a aquellos casos de infarto cerebral sin causa determinada después de una investigación etiológica adecuada. El mecanismo embólico imprime cambios característicos en el patrón de las imágenes cerebrales. El término ESUS se perfila como una entidad terapéuticamente relevante en aquellos pacientes con ataque cerebrovascular criptogénico y alta sospecha de cardioembolia que pueden beneficiarse con el uso de anticoagulantes orales directos.

La evidencia disponible para iniciar anticoagulación es persuasiva y la certeza solo se obtiene al documentar la fibrilación auricular. La monitorización cardiaca prolongada mediante dispositivos externos o insertables ha permitido detectar hasta diez veces más la fibrilación auricular paroxística que la monitorización convencional.

El rol de la fibrilación auricular paroxística en la enfermedad vascular cerebral aún no está plenamente entendido y hay evidencia creciente sobre el papel de riesgos emergente.

Por último, la relación entre cardiopatía auricular fibrótica, fibrilación auricular y trombogénesis debe establecerse con precisión.

\section{Conflicto de intereses}

Los autores declaran no tener conflicto de intereses.

\section{Bibliografía}

1. Camm AJ. Cryptogenic stroke - can we abandon this apologetic diagnosis? Nat Rev Cardiol. 2014;11:504-5.

2. Fonseca AC, Ferro JM. Cryptogenic stroke. Eur J Neurol. 2015;0:1-6.

3. Kolominsky-Rabas PL, Weber M, Gefeller O, et al. Epidemiology of Ischemic Stroke Subtypes According to TOAST Criteria Incidence, Recurrence, and Long-Term Survival in Ischemic Stroke Subtypes: A Population-Based Study. Stroke. 2001;32:2735-40.

4. Liberman AL, Prabhakaran S. Cryptogenic stroke: how to define it? How to treat it? Curr Cardiol Rep. 2013;17:423.

5. Adams HP Jr, Bendixen BH, Kappelle LJ, et al. Classification of subtype of acute ischemic stroke. Definitions for use in a multicenter clinical trial. TOAST. Trial of Org 10172 in Acute Stroke Treatment. Stroke. 1993;24:35-41.

6. Meschia JF, Barrett KM, Chukwudelunzu F, et al. Interobserver Agreement in the Trial of Org 10172 in Acute Stroke Treatment Classification of Stroke Based on Restrospective Medical Record Review. J Stroke Cerebrovasc Dis. 2006;15:266-72.

7. Ay H, Furie KL, Singhal A, et al. An evidence-based causative classification system for acute ischemic stroke. Ann Neurol. 2005;58:688-97.

8. Amarenco P, Bogousslavsky J, Caplan LR, et al. New Approach to Stroke Subtyping: The A-S-C-O (Phenotypic) Classification of Stroke. Cerebrovasc Dis. 2009;27:502-8.
9. Hart RG, Diener HC, Coutts SB, et al. Embolic strokes of undetermined source: the case for a new clinical construct. Lancet Neurol. 2014;13:429-38.

10. Bang OY, Ovbiagele B, Kim JS. Evaluation of cryptogenic stroke with advanced diagnostic techniques. Stroke. 2014;45:1186-94.

11. Fujimoto S, Toyoda K, Jinnouchi J, et al. Differences in diffusion-weighted image and transesophageal echocardiographical findings in cardiogenic, paradoxical and aortogenic brain embolism. Cerebrovasc Dis. 2011;32:148-54.

12. Jauss $M$, Wessels $T$, Trittmacher $S$, et al. Embolic lesion pattern in stroke patients with patent foramen ovale compared with patients lacking an embolic source. Stroke. 2006; 37:2159-61.

13. Jickling GC, Stamova B, Ander BP, et al. Prediction of cardioembolic, arterial, lacunar causes of cryptogenic stroke by gene expression and infarct location. Stroke. 2012;43:2036-41.

14. Scullen TA, Monlezun DJ, Siegler JE, et al. Cryptogenic stroke: clinical consideration of a heterogeneous ischemic subtype. J Stroke Cerebrovasc Dis. 2015;24:993-9.

15. Hart RG, Pearce LA, Aguilar MI. Meta-analysis: antithrombotic therapy to prevent stroke in patients who have nonvalvular atrial fibrillation. Ann Intern Med. 2007;146:857-67.

16. Verheugt FW, Granger CB. Oral anticoagulants for stroke prevention in atrial fibrillation: current status, special situations, and unmet needs. Lancet. 2015;386:303-10.

17. Kamel H, Johnson DR, Hegde M, et al. Detection of atrial fibrillation after stroke and the risk of recurrent stroke. J Stroke Cerebrovasc Dis. 2012;21:726-31.

18. Glotzer TV, Ziegler PD. Cryptogenic stroke: Is silent atrial fibrillation the culprit? Heart Rhythm. 2015;12:234-41.

19. Sanna T, Diener H-C, Passman RS, et al. Cryptogenic stroke and underlying atrial fibrillation. N Engl J Med. 2014;370:2478-86.

20. Gladstone DJ, Spring M, Dorian P, et al. Atrial fibrillation in patients with cryptogenic stroke. N Engl J Med. 2014;370: 2467-77.

21. Sposato LA, Cipriano LE, Saposnik G, et al. Diagnosis of atrial fibrillation after stroke and transient ischaemic attack: a systematic review and meta-analysis. Lancet Neurol. 2015;14: 377-87.

22. Gladstone DJ, Dorian P, Spring M, et al. atrial premature beats predict atrial fibrillation in cryptogenic stroke. Results from the EMBRACE Trial. Stroke. 2015;46:936-41.

23. Kernan WN, Ovbiagele B, Black HR, et al. Guidelines for the prevention of stroke in patients with stroke and transient ischemic attack. A guideline for healthcare professionals from the American Heart Association/American Stroke Association. Stroke. 2014:45:2160-236.

24. Healey JS, Connolly SJ, Gold MR, et al. Subclinical atrial fibrillation and the risk the risk of stroke. N Engl J Med. 2012;366:120-9.

25. Favilla CG, Ingala E, Jara J, et al. Predictors of Finding Occult Atrial Fibrillation After Cryptogenic Stroke. Stroke. 2015;46:1210-5.

26. Tayal AH, Tian M, Kelly KM, et al. Atrial fibrillation detected by mobile cardiac outpatient telemetry in Cryptogenic TIA or stroke. Neurology. 2008;71:1696-701.

27. Daoud EG, Glotzer TV, Wyse DG, et al. Temporal relationship of atrial tachyarrhythmias, cerebrovascular events, and systemic emboli based on stored device data: A subgroup analysis of TRENDS. Heart Rhythm. 2011;8:1416-23.

28. Brambatti M, Connolly SJ, Gold MR, et al. Temporal relationship between subclinical atrial fibrillation and Embolic events. Circulation. 2014;129:2094-9.

29. Ip J, Waldo AL, Lip GYH, et al. Multicenter randomized study of anticoagulation guided by remote rhythm monitoring in patients With implantable cardioverter-defibrillator and CRT-D devices: Rationale, design, and clinical characteristics of the initially enrolled cohort: The IMPACT study. Am Heart J. 2009;158, 36470.e1. 
30. Yaghi S, Boehme AK, Hazan R, et al. Atrial cardiopathy and cryptogenic stroke: a cross-sectional pilot study. J Stroke Cerebrovasc Dis. 2016;25:110-4.

31. Longstreth WT, Kronmal RA, Thompson JLP, et al. Amino Terminal Pro-B-Type natriuretic peptide, secondary stroke prevention, and choice of antithrombotic therapy. Stroke. 2013;44:714-9.

32. Yaghi S, Moon YP, Mora-McLaughlinn C, et al. Left atrial enlargement and stroke recurrence the northern Manhattan stroke study. Stroke. 2015;46:1488-93.

33. Hirsh BJ, Copeland-Halperin RS, Halperin JL. Fibrotic atrial cardiomyopathy, atrial fibrillation, and thromboembolism.
Mechanistics Links and Clinical Inferences. J Am Coll Cardiol. 2015;65:2239-51.

34. Daccarett M, Badger TJ, Akoum N, et al. Association of left atrial fibrosis detected by delayed-enhancement magnetic resonance imaging and the risk of stroke in patients with atrial fibrillation. J Am Coll Cardiol. 2011;57:831-8.

35. Marrouche NF, Wilber D, Hindricks G, et al. Association of atrial tissue fibrosis identified by Delayed enhancement MRI and Atrial Fibrillation Catheter Ablation The DECAAF Study. JAMA. 2014;311:498-506. 